\title{
Massive cerebral venous sinus thrombosis in vaccine-induced immune thrombotic thrombocytopenia after ChAdOx1 nCoV-19 serum: case report of a successful multidisciplinary approach
}

\author{
Laura Mirandola ${ }^{1}$. Giulia Arena ${ }^{2} \cdot$ Maria Pagliaro $^{3}$ - Andrea Boghi ${ }^{4} \cdot$ Andrea Naldi $^{1}$. Davide Castellano ${ }^{4}$. \\ Antonella Vaccarino ${ }^{3} \cdot$ Daniela Silengo $^{2} \cdot$ Franco Aprà $^{5} \cdot$ Roberto Cavallo $^{1} \cdot$ Sergio Livigni ${ }^{2}$
}

Received: 27 October 2021 / Accepted: 16 November 2021 / Published online: 10 January 2022

(c) Fondazione Società Italiana di Neurologia 2021

\begin{abstract}
We report a case of massive cerebral venous sinus thrombosis in the contest of vaccine-induced immune thrombotic thrombocytopenia that required the rapid coordination of many specialists from different departments, notably emergency, neurology, neuroradiology, hematology, and neurosurgery. The patient was rapidly treated with steroids, immunoglobulin, and fondaparinux. She underwent within $6 \mathrm{~h}$ after hospital admission a mechanical thrombectomy in order to allow flow restoration in cerebral venous systems. Neuroendovascular treatment in cerebral venous thrombosis related to VITT has never been described before. It can represent a complementary tool along with the other therapies and a multidisciplinary approach.
\end{abstract}

Keywords VITT $\cdot$ Immunotherapy $\cdot$ Anticoagulation $\cdot$ Thrombectomy $\cdot$ Multimodal monitoring

\section{Background}

Vaccine-induced immune thrombotic thrombocytopenia (VITT) is a recently recognized rare syndrome characterized by immune-mediated thrombosis in typical and atypical sites, combined with mild to severe thrombocytopenia and positive antibodies against platelet factor 4 (PF4), in individuals recently vaccinated with adenoviral vector vaccine against Covid-19 [1-4]. VITT incidence is not established, with an estimate rate of approximately 1 case per 125,000 for ChAdOx1 nCoV-19 vaccine (Oxford-Astra-Zeneca) and

Laura Mirandola

laura.mirandola05@gmail.com

1 SC Neurologia, Ospedale San Giovanni Bosco, ASL Città Di Torino, Turin, Italy

2 SC Anestesia E Rianimazione 2, Ospedale San Giovanni Bosco, ASL Città Di Torino, Turin, Italy

3 SSD Ematologia, Ospedale San Giovanni Bosco, ASL Città Di Torino, Turin, Italy

4 SC Radiologia E Neuroradiologia, Ospedale San Giovanni Bosco, ASL Città Di Torino, Turin, Italy

5 SC MECAU 2, Ospedale San Giovanni Bosco, ASL Città Di Torino, Turin, Italy
1 per 1,000,000 for the Ad26.Cov2.S vaccine (Johnson and Johnson) [3].

Several expert guidelines for the evaluation and management of VITT have been recently produced, such as the Italian Society for the study of Hemostasis and Thrombosis (SISET) [5, 6].

Here, we describe the case of a patient who developed VITT with massive cerebral venous sinus thrombosis after ChAdOx $1 \mathrm{nCoV}-19$ vaccine treated with a multidisciplinary approach resulting in a complete recovery.

\section{Case presentation}

A 52-year-old Caucasian woman was admitted to emergency department for a suspected stroke. Her past medical history was unremarkable. She was on therapy with oral contraception. She had the first dose of ChAdOx1 nCov-19 vaccine 15 days before. Sickness, nausea, and vomiting were reported since the previous day. At arrival, she was unresponsive, with head and eyes deviation to the right and left hemiparesis. The vital parameters were normal. No other signs of thrombosis or bleeding were noticed at physical examination. Sars-Cov2 rapid and molecular nasopharyngeal tests were both negative. 
Head CT scan revealed diffuse brain edema and spontaneous hyperdensity in the superior sagittal sinus and the straight sinus according with a diagnosis of massive cerebral venous sinus thrombosis (CVST); CT angiography (CTA) confirmed a diffuse CVST (Fig. 1a). A total body CECT scan ruled out venous thrombosis in other sites.
Blood tests revealed a severe isolated thrombocytopenia $(40.000 / \mathrm{mmc})$ and a slight D-dimer increase $(18 \mathrm{mcg} / \mathrm{ml})$.

Due to the clinical suspect of VITT, dexamethasone (40 mg i.v. daily for 4 days), high-dose immunoglobulins ( $1 \mathrm{~g} / \mathrm{kg}$ body weight daily for 2 days i.v.), and low dose of fondaparinux ( $2.5 \mathrm{mg}$ subcutaneously) were administered in less than an hour since hospital admission (Fig. 2),
Fig. 1 A Head CT scan/CT angiography. On the left axial CT section showing diffuse brain edema and hyperdensity in the superior sagittal sinus, on the right $\mathrm{CT}$ angiography confirming massive cerebral venous sinus thrombosis involving superior sagittal sinus, torcular, the right dominant transverse and sigmoid sinuses, the straight sinus, the proximal portion of the right internal jugular vein and several convexity hemispheric veins of the fronto-parietal region bilaterally adjacent to the superior sagittal sinus. B Brain MR imaging. Axial sections of brain MRI, on the left susceptibility waited imaging (SWI) sequence and on the right left fluid attenuated inversion recovery (FLAIR) sequence showing edema and microbleeds in right perirolandic cortex, without sign of restricted diffusion, meaningful of venous engorgement edema. C Brain angiography at the end of the neuroendovascular treatment showing a complete patency of the superior sagittal sinus, torcular and right transverse and sigmoid sinuses with residual thrombi in cortical veins. The straight sinus was not completely reopened, but a small channel draining the internal cerebral veins was obtained



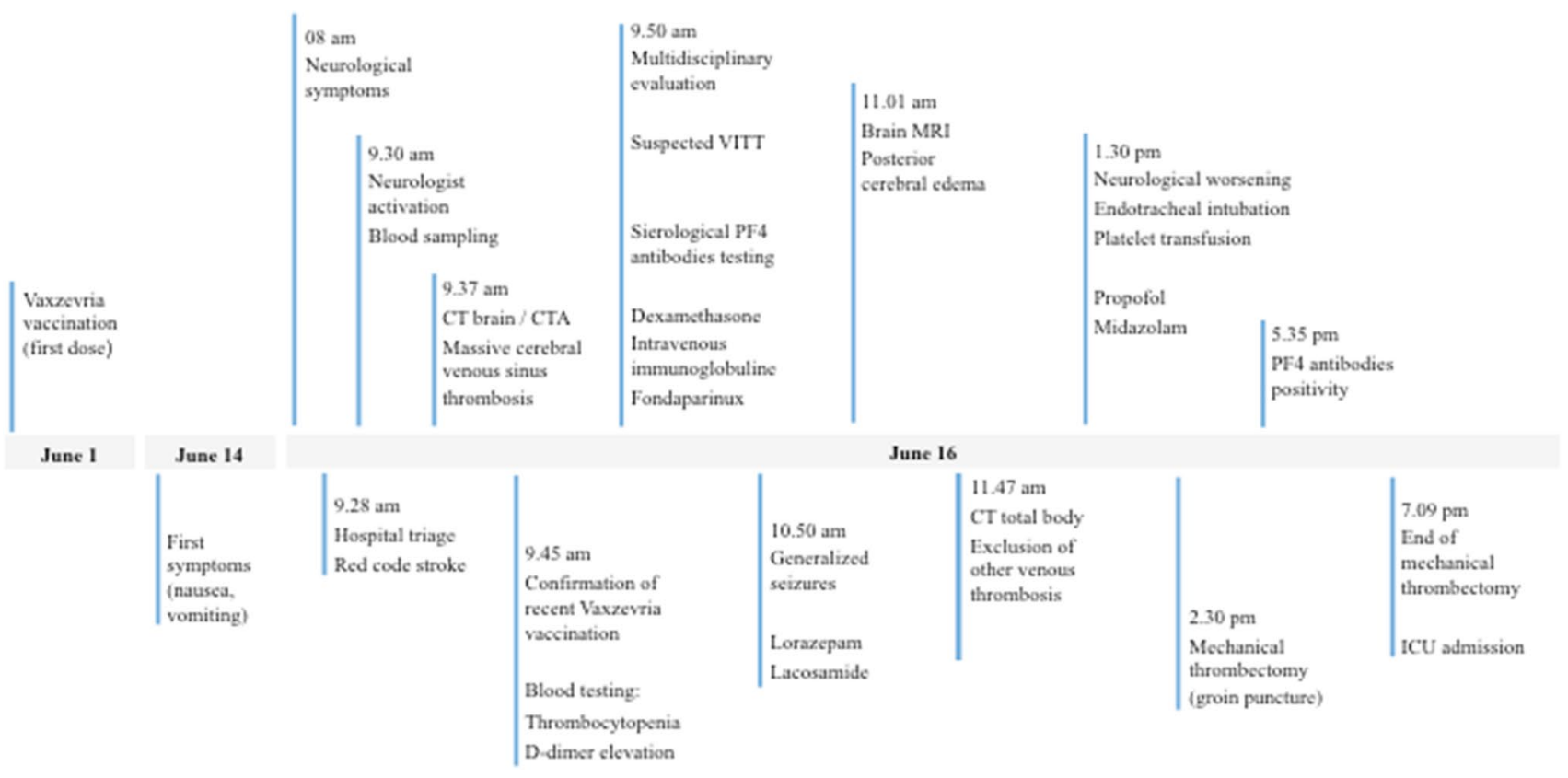

Fig. 2 Time line. This diagram shows the temporal evolution from vaccination date to ICU admission

according to SISET guidelines. In the following hours, IgG against PF4/heparin (ELISA) and functional platelet aggregation test (PAT heparin-induced platelet aggregation) resulted positive, thus confirming the diagnosis of VITT. A subsequent brain MR imaging confirmed the diffuse cerebral venous thrombosis (Fig. 1b). At the end of the MRI, after a tonic-clonic seizure, the patient experienced progressive neurological deterioration; therefore, she was intubated. The neuroradiologist suggested a neuroendovascular treatment and after a collegial discussion, we decided to proceed after platelet transfusion. Thrombectomy was performed both with aspiration using large bore aspiration catheter and with multiple embricated stent-retrievers. After many passages, the superior sagittal sinus, torcular, and right transverse and sigmoid sinuses were recanalized with residual thrombi in cortical veins. The straight sinus was not completely reopened, however obtaining a small channel draining the internal cerebral veins. The mechanical thrombectomy allowed flow restoration both in superficial and deep cerebral venous systems but not in cortical veins (Fig. 1c). At the end of the procedure, day 0 , the patient was admitted in ICU where she stayed for 12 days. A new brain and chest CT scan excluded bleeding and malignant brain edema. We decided to place intraparenchymal monitoring of intracranial pressure (ICP) before starting full-dose anticoagulation. Frequent periodic epileptic activity was recorded on continuous electroencephalographic monitoring; therefore, antiepileptic treatment was increased (Levetiracetam $3 \mathrm{~g}$ daily in association with Lacosamide $400 \mathrm{mg}$ daily). Since day 1, platelet count starts increasing and fully recovered on day 9 . Starting from day
2 , in agreement with the hematologist, incremental dosage of fondaparinux was set up until full anticoagulant dose of fondaparinux (7.5 mg s.c./die). Screening tests for congenital and acquired thrombophilia were normal.

MRI was repeated on day 11 , showing complete patency of the superior sagittal, torcular, and left transverse and sigmoid sinuses, whereas the right transverse and sigmoid sinuses were again occluded. Both internal cerebral veins, the vein of Galen and the straight sinus, were patent, although with a faint flow. Brain edema was regressed whereas signs of microbleeds in right perirolandic cortex and right thalamus were unchanged.

Progressive neurological improvement was observed until recovery of consciousness on day 9 ; on day 12 , the patient was extubated and transferred to Neurology Unit. After 22 days, the patient returned home with a complete neurological recovery. She was discharged with antiepileptic drugs under reduction and warfarin with a target INR between 2 and 3 .

\section{Discussion and conclusion}

Cerebral venous sinus thrombosis is highly associated to VITT and can be responsible of severe morbidity and mortality [4, 7]. Before the recent descriptions of VITT [1-7], the management of such a massive CVST would have included continuous i.v. heparin immediately after CT scan, which is contraindicated in the management of VITT because of the presence of antiEPF4 antibodies [1]. Due to 
the immunological pathogenesis of VITT, we believe it is important to suspect VITT and to propose a rapid administration of immunotherapy together with anticoagulation different from heparin. In our case, we decided to use fondaparinux in accordance with the SISET guidelines [5, 6]. Even if we found some reported experiences using DOAC in the treatment of cerebral veins thrombosis [8], upon discharge, we decided to proceed with a "conventional" longterm anticoagulation with warfarin, the only available in label in Italy.

Anticoagulant therapy is considered the first-line therapy for CVST, in order to prevent thrombosis progression and to re-establish venous flow [5]. In our patient, CVST was massive, involving both superficial and deep venous systems, so the medical therapy alone would probably have not been enough to resolve the intracranial venous congestion. Therefore, the endovascular intervention together with anticoagulant therapy has been recommended for our experience despite very low quality of available evidence and the clinical worsening due to the massive cerebral sinus thrombosis, frequently associated to a poor prognosis [9].

In our patient, the thrombectomy with medical treatments and multimodal intensive care monitoring, for rapid recognition and treatment of complication, ended in an excellent clinical outcome.

\section{Declarations}

Conflict of interest The authors declare no competing interests.

Ethical approval The study was performed in accordance with the ethical standards as laid down in the 1964 Declaration of Helsinki and its later amendments or comparable ethical standards.

Informed consent Informed written consent was obtained from the patient.

\section{References}

1. Handtke S, Wolff M, Zaninetti C et al (2021) A flow cytometric assay to detect platelet-activating antibodies in VITT after ChAdOx1 nCov-19 vaccination. Blood 137(26):3656-3659

2. Schultz NH, Sørvoll IH, Michelsen AE et al (2021) Thrombosis and thrombocytopenia after ChAdOx1 $\mathrm{nCoV}-19$ vaccination. $\mathrm{N}$ Engl J Med 384:2124-2130

3. Schulz JB, Berlit P, Diener HC et al (2021) COVID-19 vaccineassociated cerebral venous thrombosis in Germany. Ann Neurol 90(4):627-639

4. Greinacher A, Thiele T, Warkentin TE et al (2021) Thrombotic thrombocytopenia after ChAdOx1 $\mathrm{nCov}-19$ vaccination. $\mathrm{N}$ Engl J Med 384:2092-2101

5. Gresele P, Marietta M, Ageno W et al (2021) Management of cerebral and splanchnic vein thrombosis associated with thrombocytopenia in subjects previously vaccinated with Vaxzevria (AstraZeneca): a position statement from the Italian Society for the Study of Haemostasis and Thrombosis (SISET). Blood Transfus 19:281-283

6. Franchini M, Liumbruno GM, Pezzo M (2021) COVID-19 vaccine-associated immune thrombosis and thrombocytopenia (VITT): diagnostic and therapeutic recommendations for a new syndrome. Eur J Haematol 107(2):173-180

7. Scully M, Singh D, Lown R et al (2021) Pathologic antibodies to platelet factor 4 after ChAdOx1 nCoV-19 vaccination. N Engl J Med 384:2202-2211

8. Guetl K, Gary T, Raggam RB et al (2021) SARS -COV-2 vaccine induce immune thrombotic thrombocytopenia treated with immunoglobulin and argatroban. Lancet 397(10293):e19

9. Coutinho JM, Zuurbier SM, Bousser MG et al (2020) Effect of endovascular treatment with medical management vs standard care on severe cerebral venous thrombosis: the TO-ACT randomized clinical trial. JAMA Neurol 77(8):966-973

Publisher's note Springer Nature remains neutral with regard to jurisdictional claims in published maps and institutional affiliations. 